\title{
NUMERICAL STUDY ON SUPPRESSION OF TSUNAMI WITH UNDER-SEA BREAKWATER
}

\author{
HISASHI HORIUCHI' ${ }^{1}$ AND MAKOTO YAMAMOTO \\ ${ }^{1}$ Graduate School of Mechanical Engineering, Tokyo University of Science \\ 6-3-1 Niijyuku, Katsushika-ku, Tokyo, Japan \\ E-mail: j4513647@ed.tus.ac.jp \\ ${ }^{2}$ Department of Mechanical Engineering, Tokyo University of Science \\ 6-3-1 Niijyuku, Katsushika-ku, Tokyo, Japan \\ Email: yamamoto@rs.kagu.tus.ac.jp
}

Key words: Tsunami, MPS method, Under-sea breakwater, Wave generator.

\begin{abstract}
Large-scale tsunami occurs every 100 or 150 years statistically, and $95 \%$ of these tsunami lead to great disasters which kill over 1,000 people. In order to avoid, suppress or mitigate such damage, it is necessary to perform a risk management from the point of view of disaster prevention engineering. There are breakwaters as one of existing measures for tsunami, and the effectiveness of breakwaters has been demonstrated. However, a conventional breakwater can be disrupted by the impact of tsunami, and then it turns into debris that causes to expand the damage. There is also the problem of impairing the landscape by locating a large-scale breakwater along a sea shore. Construction of under-sea breakwaters is one possible way to coping with these problems. In this paper, tsunami behavior when the under-sea breakwater is located is numerically simulated using MPS method, which is one of the particle methods. And the effectiveness on suppression of tsunami with the under-sea breakwater is researched and verified. Through the present study, it was confirmed that the under-sea breakwater can suppress tsunami. The higher the under-sea breakwater is, the more it suppresses tsunami. However, if the height of the breakwater is too low, the effect on tsunami suppression is small. In addition, it was found that, by setting under-sea breakwater at a suitable position, arrival time of a tsunami can be delayed.
\end{abstract}

\section{INTRODUCTION}

The Great East Japan Earthquake which occurred on March 11, 2011 caused a devastating tsunami. The giant tsunami that devastated Tohoku area on the Pacific coast killed over 15,000 people and more than 3,000 people are still missing. A breakwater is one of the effective counter-measure against tsunami disasters, however, the tsunami attacked Tohoku area was so high that it run over the breakwater built based on the knowledge of the tsunami disaster in the past, and many breakwater was disrupted by the impact of tsunami. Main factors that make the breakwater disrupted are the seabed erosion and extremely large horizontal force. Difference in water level between the inside and outside of the breakwater generates the horizontal force that presses the breakwater, and then, the overtopping wave and fast flow that pass through a joint of the breakwater entrench the seabed. Due to these factors, 
the breakwater blocks slipped down from the seabed. Then, the flow converge on the location which the block slipped out, and advance of seabed-erosion leads other blocks on the seabed to slip out.

In this way, a conventional breakwater can be disrupted by the unexpected great tsunami. This problem may be solved to heighten a breakwater. But it is not a realistic way to build excessively high breakwater because of enormous cost and impairing the landscape. Thus it is necessary to propose a new breakwater technique alternative to a conventional breakwater. An under-sea breakwater is one possible way to coping with these problems.

An under-sea breakwater is one of the breakwaters, and the whole structure is within the sea. Advantages of an under-sea breakwater are: (1) The horizontal force generated by the sea-level difference is not so large, because an under-sea breakwater is located offshore. (2) An under-sea breakwater doesn't impair the landscape. However, since an under-sea breakwater does not have enough height to completely prevent tsunami from coming in the port, it is necessary to verify how effective on suppression of tsunami an under-sea breakwater is.

In the previous studies, the numerical approaches to tsunami behavior on a conventional breakwater have been conducted. Especially, in these days, Lagrangian particle methods have been used for solving the flow fields associated complicated boundary shapes like tsunami. In Lagrangian approaches, particles move in Lagrangian coordinates and the advection is directly calculated by particle motion without numerical diffusion that usually encountered in the Eulerian grid methods. It is well known that the numerical diffusion becomes a severe problem when the deformation of free surface is very large.

Koshizuka et al. ${ }^{[1]}$ indicated that the moving particle semi-implicit (MPS) which was developed for incompressible flow with free surfaces, agreed with the experimental result, even if the fragmentation and coalescence of the fluid occurred. Gotoh et al. ${ }^{[2]}$ showed that the MPS method has successfully been applied to the wave breaking and overtopping process in front of an upright seawall, through comparisons with the results of hydraulic experiments. Lee et al. ${ }^{[3]}$ confirmed that the MPS method was reasonably applied to a numerical simulation of predicting the efficiency of floating-type breakwater interacting with waves.

However, the effectiveness of under-sea breakwaters has not been investigated so far. Therefore, in this study, tsunami behavior when the under-sea breakwater is located in twodimensional area is numerically simulated, using MPS method. And, the effectiveness on suppression of tsunami influenced by changing the height and location of the under-sea breakwater is researched. We confirmed that an under-sea breakwater is effective in suppressing tsunami.

\section{NUMERICAL METHOD}

\subsection{Governing function}

In the MPS method proposed by Koshizuka et al. ${ }^{[1]}$, each term of governing equations is discretized as an interaction between particles, or calculation points, which are arranged in a computational domain. The governing equations for incompressible viscous flows are the continuity and Navier-Stokes equations, as follows: 


$$
\begin{gathered}
\frac{D \rho}{D t}=0 \\
\frac{D \mathbf{u}}{D t}=-\frac{1}{\rho} \nabla P+\downarrow \nabla^{2} \mathbf{u}+\mathbf{F}
\end{gathered}
$$

where $\rho$ is the density, $t$ is the time, $\mathbf{u}$ is the velocity, $\nabla$ is the gradient, $P$ is the pressure, $v$ is the kinematic viscosity and $\mathbf{F}$ is the external force respectively. The left side of equation (2) is the Lagrangian time differentiation involving advection terms. In a MPS method the advection terms are directly incorporated into the calculation by moving particles.

\subsection{Kernel function}

The governing equations written with partial differentiations are transformed to the equation for particle interactions. The particle interactions with its neighboring particles covered with a weight function $w(r)$, where $r$ is a distance between two particles. In this study, the weight function is followed as:

$$
w(r)=\left\{\begin{array}{cc}
\left(\frac{r_{e}}{r}-1\right)^{2} & \left(0 \leq r<r_{e}\right) \\
0 & \left(r_{e}<r\right)
\end{array}\right.
$$

where $r_{e}$ represents the effective radius of the particle interactions. The weight function is described by kernel function, i.e, the nearer the distance between two particles is, the larger the weight of the interactions is. If the distance between two particles is significantly far, the interactions can be neglected. Thus a finite number of neighboring particles are related to the interactions.

When a particle $i$ and its neighbors $j$ are located at $\mathbf{r}_{i}$ and $\mathbf{r}_{j}$, particle number density is defined as:

$$
n_{i}=\sum_{j \neq i} w\left(\left|\mathbf{r}_{j}-\mathbf{r}_{i}\right|\right)
$$

In incompressible flow, fluid density is required to be constant, and thus the particle number density is constant. In this study, the constant value of the particle number density is denoted by $n^{0}$.

\subsection{Gradient and Laplacian model}

Governing equation is discretized to use a gradient model and a Laplacian model as follows:

$$
\begin{gathered}
\langle\nabla \phi\rangle_{i}=\frac{d}{n^{0}} \sum_{j \neq i}\left[\frac{\phi_{j}-\phi_{i}}{\left|\mathbf{r}_{j}-\mathbf{r}_{i}\right|}\left(\mathbf{r}_{j}-\mathbf{r}_{i}\right) w\left(\left|\mathbf{r}_{j}-\mathbf{r}_{i}\right|\right)\right] \\
\left\langle\nabla^{2} \phi\right\rangle_{i}=\frac{2 d}{n^{0} \lambda} \sum_{j \neq i}\left(\phi_{j}-\phi_{i}\right) w\left(\left|\mathbf{r}_{j}-\mathbf{r}_{i}\right|\right)
\end{gathered}
$$

where $\phi$ is a physical quantity and $d$ is the number of space dimensions. $\lambda$ is a parameter 
where the increase in the variance is equal to that of the analytical solution given by:

$$
\lambda=\sum_{j \neq i} w\left(\left|\mathbf{r}_{j}-\mathbf{r}_{i}\right|\right) \mathbf{r}_{j}-\left.\mathbf{r}_{i}\right|^{2} / \sum_{j \neq i} w\left(\left|\mathbf{r}_{j}-\mathbf{r}_{i}\right|\right)
$$

Equation (5) means that the gradients between two adjacent particles are summed, considering the weight function. Equation (6) means that physical property of the target particle is distributed onto the particles in its neighborhood. General concepts of these models are shown in Fig. 1.
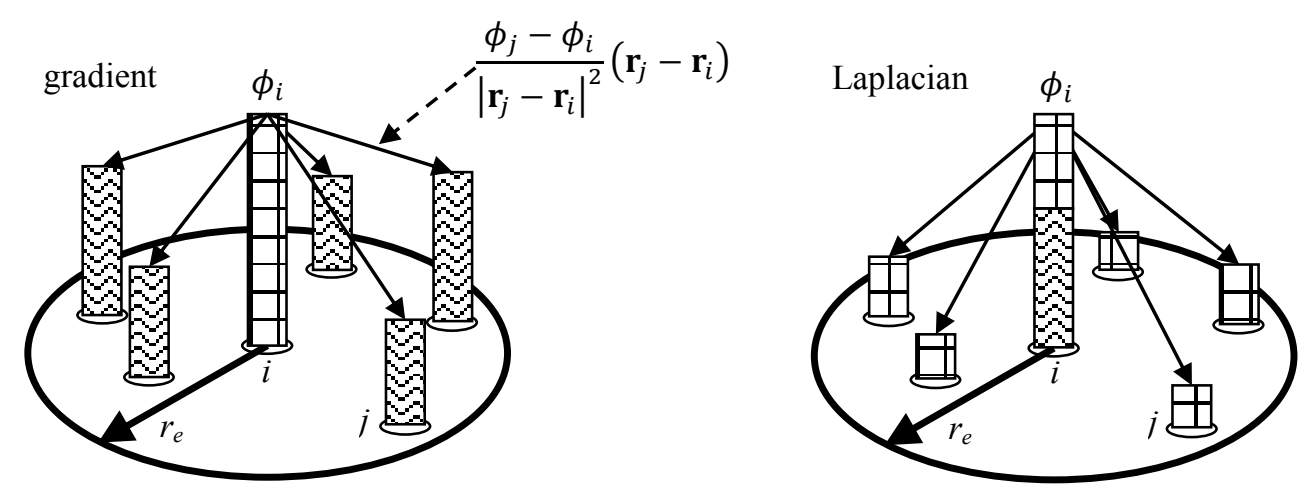

Figure 1: Concepts of gradient and Laplacian models

\subsection{Modeling of incompressibility}

There are two stages in each time step. In the first stage, the temporal velocity components and coordinates of the particle $i$ are obtained using diffusion, external forces and convection terms, which are explicitly calculated in the $(n)$-th time step. Then the temporal coordinates $\left(\mathbf{r}_{i}{ }^{*}\right)$ can be calculated using the temporal velocity $\mathbf{u}_{i}{ }^{*}$, expressed by:

$$
\mathbf{r}_{i}^{*}=\mathbf{r}_{i}^{n}+\Delta t \mathbf{u}_{i}^{*}
$$

Due to the movement of particles in the explicit first stage, the particle number densities might be changed. In the second stage, the pressure term which was not considered in the first stage is taken into account, and the Poisson equation of pressure is implicitly solved to update the pressure field as follows:

$$
\left\langle\nabla^{2} P\right\rangle_{i}=-\frac{\rho}{\Delta t^{2}} \frac{n_{i}^{*}-n^{0}}{n^{0}}
$$

where $\Delta t$ means the time increment of the calculation. The right side of Equation (9) is the deviation of the particle number density, and it corresponds to the divergence of velocity vector in a finite difference method. The left side of Equation (9) is discretized to simultaneous linear equations, using the present Laplacian model, Equation (6). The simultaneous linear equations are solved using the incomplete Cholesky decomposition conjugate gradient (ICCG) method. 


\subsection{Boundary conditions}

The wall boundary is made up of several rows of fixed particles arranged regularly. For the particle number density calculation, the thickness of wall must be set thicker than the threshold of the weight function, $r_{e}$. The condition of velocity, such as the non-slip condition, is given on the wall surface particles touching directly with fluid, on which the location is not updated, and only the pressure is updated. The judgment of the water surface is carried out based on the condition of the particle number density as follows:

$$
n_{i}^{*}<\beta \cdot n^{0}
$$

where $\beta$ is a existential parameter, in this study $\beta=0.97$, following Koshizuka and $\mathrm{Oka}^{[1]}$. A boundary condition of the pressure $P=0$ is given on the water surface particles. This boundary condition, which does not depend on the configuration of the water surface, is easily applied to the complex shape of the water surface, such as the fragmentation of water. On particles isolated by splash due to the water fragmentation, the interaction terms with neighborhood particles are neglected. In other words, the isolated particles fall in the gravity field under the initial condition of velocity, or the particle velocity at the time of isolation.

In this study, different sizes of the weight function are used. The size which is used for the particle number density and the gradient model is $r_{e}=2.1 l_{o}$, where $l_{o}$ is the distance between two adjacent particles in the initial configuration. The value of 2.1 was selected to avoid the concentration of particles near the free surfaces. On the other hand, the size which is used for the Laplacian model is $r_{e}=4.0 l_{o}$. The value of 4.0 was selected by the balance between computation time and accuracy. These parameters are also followed by Koshizuka and Oka ${ }^{[1]}$.

\section{COMPUTATIONAL CONDITIONS}

In this study, the calculation domain is the simplified seabed topography. The geometry is shown in Fig. 2. The waves are generated by an offshore vertical wall. The motion of wave generator is changed to follow the velocity as follows:

$$
V_{w}(t)=V_{0} \sin (2 \pi t / T)
$$

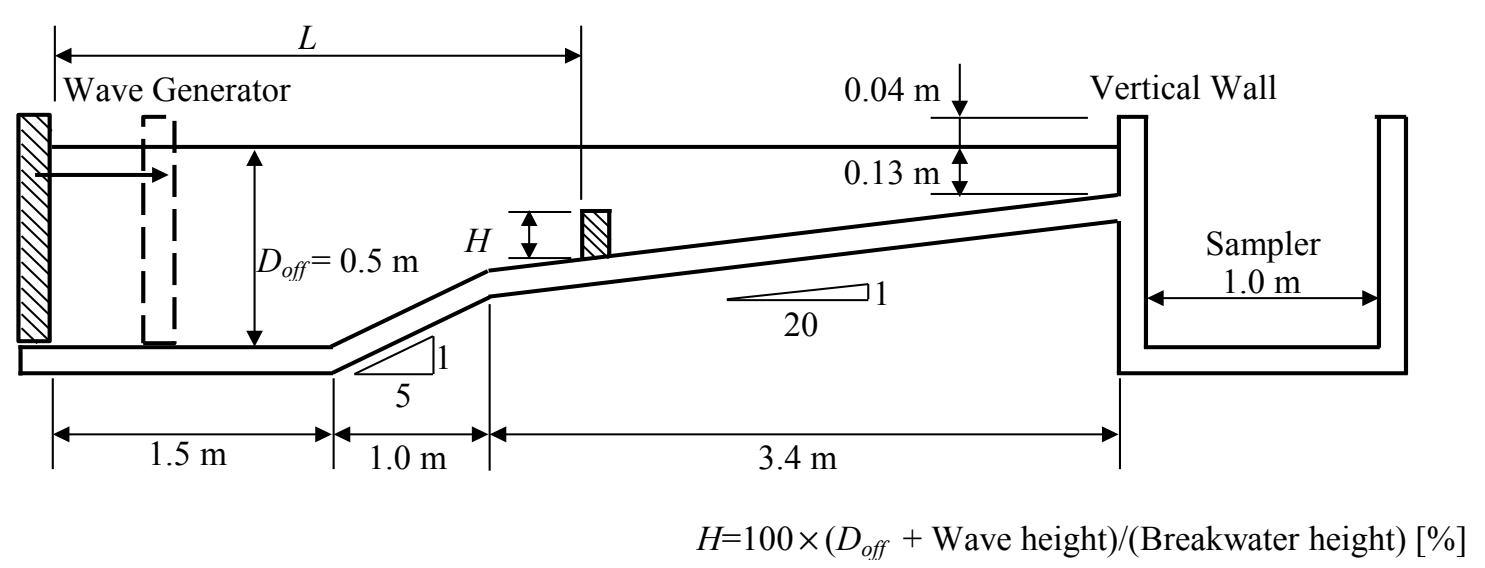

Figure 2: Computational domain and dimension 
where $T$ denotes the wave period $(=2.0[\mathrm{~s}])$ and $V_{0}$ is maximum speed of wave generator $(=0.55[\mathrm{~m} / \mathrm{s}]) . H$ is the height of under-sea breakwater. $H$ is changed from 10 to $60[\%]$ of the water depth to keep the breakwater under the sea surface. $L$ is the location of the under-sea breakwater from the wave generator and it ranges from 2.8 to $4.4 \mathrm{~m}$.

The kinetic momentums of overtopping particles over the ashore vertical wall is compared, which differ according to the height and location of under-sea breakwater. In addition, the wave height and the arrival time at the front of the ashore upright wall are measured to investigate suppression of wave with under-sea breakwater. The number of particles with particle diameter $d_{0}=0.01 \mathrm{~m}$ is approximately 25,000 . We assumed that the calculated fluid is defined as water, the density is $998.2 \mathrm{~kg} / \mathrm{m}^{3}$ and the kinetic viscosity is $1.004 \times 10^{-6} \mathrm{~m}^{2} / \mathrm{s}$.

\section{RESULTS AND DISCUSSION}

Figure 3 indicates the kinetic momentum of wave overtopping particles for the different height and location of the under-sea breakwater. The vertical axis is a percentage of a momentum normalized with the case without an under-sea breakwater. The approximated curves are calculated using a least-square method. In $H=55 \%$, the kinetic momentum of wave overtopping particles is reduced by approximately $50 \%$. In addition, in the cases that $H$ is more than $40 \%$, the higher the under-sea breakwater is, the greater suppressing effect is without regard to $L$. However, in the cases that $H$ is less than $40 \%$, some cases do not have a suppressive effect, and inversely the under-sea breakwater tends to enhance tsunami.

Figure 4 represents the water speed distribution around the under-sea breakwater which is located at $2.8 \mathrm{~m}$; (a) $H=25.0 \%$ and (b) $H=52.8 \%$. The water speed is the highest on the sea surface and decrease as closer to the sea bed. Thus, with lower under-sea breakwater, a suppressive effect of a wave motion disappears because the under-sea breakwater exist in the space where particle movement is small, and the number of particles which run over the under-sea breakwater increase. Due to the theory of continuum, wave particles accelerate over the under-sea breakwater, and the momentum of wave increases. On the other hand, with higher under-sea breakwater, the momentum of wave particles decreases because the under-

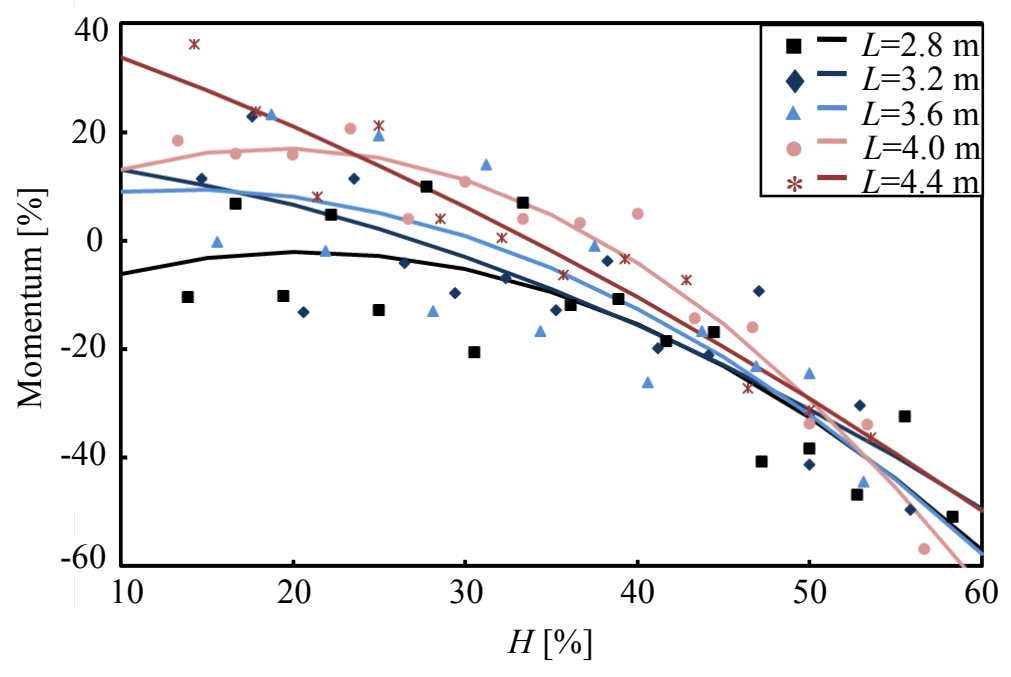

Figure 3: Momentum of overtopping wave 
sea breakwater can stop many particles in front of the breakwater even if wave particles over the breakwater accelerate more.

The wave height measured at the front of the ashore upright wall is shown in Fig. 5, when the under-sea breakwater is placed at $\mathrm{L}=2.8 \mathrm{~m}$. The wave height does not change remarkably as the height of under-sea breakwater is changed, and the correlation with the momentum variation cannot be obtained. However, the arrival time of a wave can be delayed due to the under-sea breakwater. In Fig. 4, when the under-sea breakwater height is $52.8 \%$, the wave is already delayed in the back of the under-sea breakwater as compared to the case of $H=25.0 \%$. In the case of the higher under-sea breakwater, the wave over the under-sea breakwater breaks

(a) $\mathrm{H}=25.0 \%$

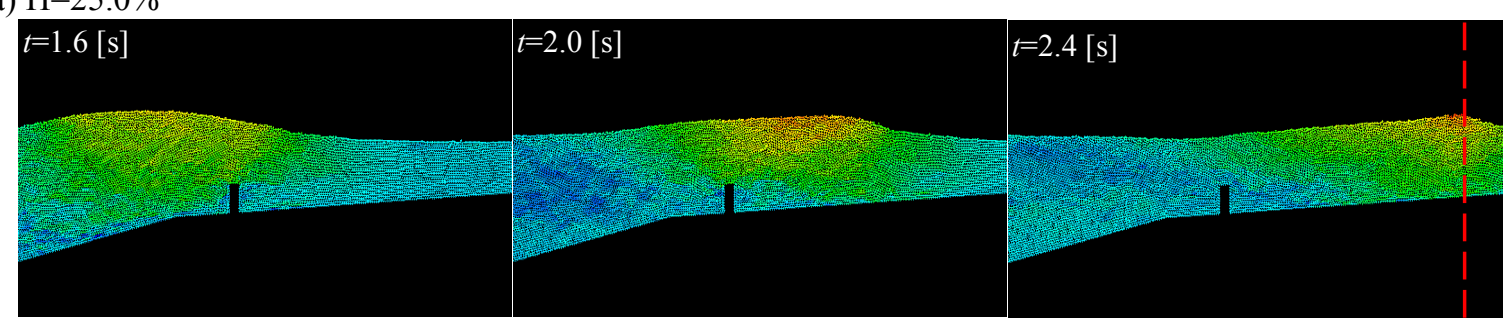

(b) $\mathrm{H}=52.8 \%$

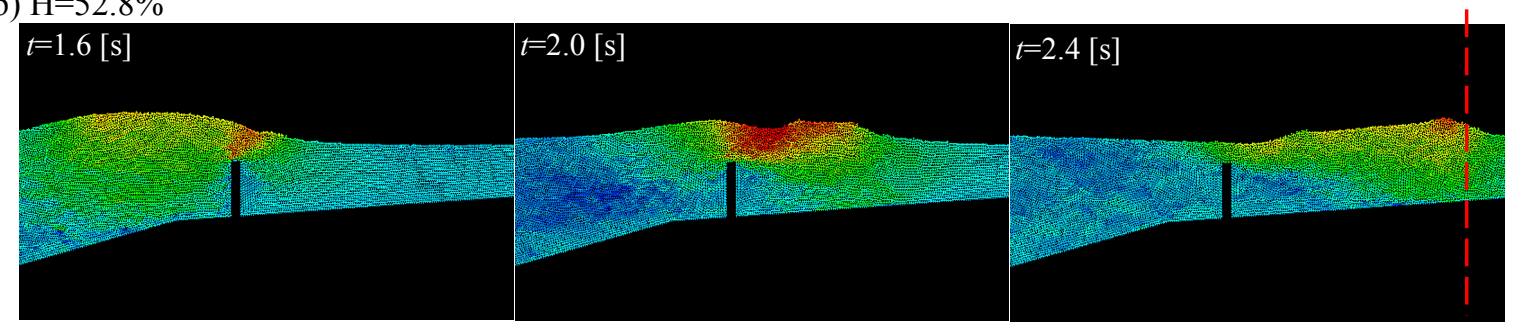

Figure 4: Water speed distribution around under-sea breakwater

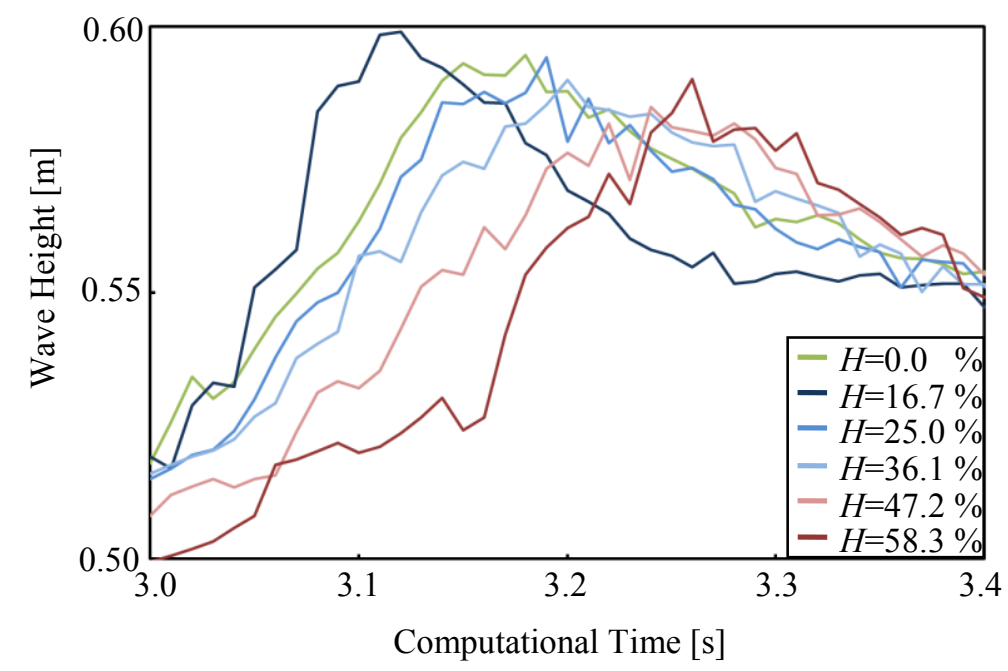

Figure 5: Wave height at front of vertical wall 
up, and then generates a new wave (Fig. 4 (b), $t=2.0 \mathrm{~s}$ ). The delayed arrival time would be caused by the lagged time to generate a new wave.

\section{CONCLUSIONS}

The prediction of the performance of an under-sea breakwater was investigated numerically, using the MPS method proposed by Koshizuka et al. ${ }^{[1]}$ for an incompressible flow.

From the simulated results, an under-sea breakwater can suppress tsunami, but it is necessary to heighten an under-sea breakwater more than $40 \%$ of the water depth. In the case that the height of the under-sea breakwater is less than $40 \%$, the under-sea breakwater cannot only suppress a kinetic momentum of wave, but also sometimes increase a momentum of wave. In addition, the arrival time of tsunami can be delayed since the wave is collapsed by a higher under-sea breakwater.

In our future work, we will clarify the three-dimensional effect of under-sea breakwaters.

\section{REFERENCES}

[1] Koshizuka, S. and Oka, Y. Moving Particle Semi-implicit Method for Fragmentation of Incompressible Fluid. Nulc. Sci. Engng. (1996) 123:421-434

[2] Gotoh, H. Ikari, H. Memita, T. and Sakai, T. LAGRANGIAN PARTICLE METHOD FOR SIMULATION OF WAVE OVERTOPPING ON A VERTICAL SEAWALL. Coast. Engng. J. (2005) 47:157-181.

[3] Lee, B.H. Hwang, S.C. Nam, J.W. and Park, J.C. Numerical prediction for the performance of a floating-type breakwater by using a two-dimensional particle method. Int. J. Oc. System Engng.(2011) 1:37-45. 